\title{
Comparison of fundus fluorescein angiography, optical coherence tomography and optical coherence tomography angiography features of macular changes in Eales disease: a case series
}

Ketaki Rajurkar* ${ }^{*}$, Meenakshi Thakar, Priyadarshi Gupta and Anju Rastogi

\begin{abstract}
Purpose: To study the macular features in Eales disease patients observed with fundus fluorescein angiography (FA), optical coherence tomography (OCT) and optical coherence tomography angiography (OCTA).

Methods: A cross-sectional study was done on treatment naïve 31 eyes (23 patients) with Eales disease. Baseline parameters such as Best-corrected visual acuity (BCVA), slit-lamp bio microscopy (SLB), indirect ophthalmoscopy, FA, spectral-domain OCT qquantitative (central macular thickness [CMT]) and qualitative analysis on SD-OCT\} and OCTA were performed. Any media opacity precluding the above investigations was excluded.

Results: Macular findings comprised of- epiretinal membrane, macular exudation, full thickness macular hole, sub internal limiting membrane bleed, cystoid macular oedema, neurosensory detachment and retinal thickening. Sixteen (51.6\%) of our patients had macular changes as seen on all modalities together. SLB and indirect ophthalmoscopy missed macular findings in 50\% patients and FA in 18.8\% patients. OCT and OCTA diagnosed all macular findings. On comparison of mean BCVA in patients with macular involvement on FA, OCT and OCTA, compared to those without macular involvement, patients with macular involvement had lower BCVA ( $p 0.000,0.01$ and 0.001 respectively). Thus, FA missed many patients who had significant macular involvement and hence less vision.

Conclusion: Eales disease though described in literature as classically being peripheral retina disease process, also has macular involvement. OCT and OCTA are useful guides to evaluation of macular involvement in these patients. The latter seems to be superior to FA in detecting macular abnormalities in this ailment. OCTA is non-invasive and shows deep capillary plexus changes which are not shown by any other modality.
\end{abstract}

Keywords: Eales disease, Slit lamp bio microscopy, Optical coherence tomography, Optical coherence tomography angiography, Deep capillary plexus

\footnotetext{
* Correspondence: k.rajurkar25@gmail.com

Guru Nanak Eye Centre, Near Zakir Hussain College, Maharaja Ranjeet Singh

Marg, 64 Khamba, New Delhi, Delhi 110002, India
}

Springer Open (c) The Author(s). 2020 Open Access This article is licensed under a Creative Commons Attribution 4.0 International License, which permits use, sharing, adaptation, distribution and reproduction in any medium or format, as long as you give appropriate credit to the original author(s) and the source, provide a link to the Creative Commons licence, and indicate if changes were made. The images or other third party material in this article are included in the article's Creative Commons licence, unless indicated otherwise in a credit line to the material. If material is not included in the article's Creative Commons licence and your intended use is not permitted by statutory regulation or exceeds the permitted use, you will need to obtain permission directly from the copyright holder. To view a copy of this licence, visit http://creativecommons.org/licenses/by/4.0/. 


\section{Introduction}

Eales disease was first described by the British ophthalmologist Henry Eales in 1880. Eales disease is an idiopathic, occlusive perivasculitis affecting the peripheral retina, leading to retinal non-perfusion, new vessel formation, and recurrent vitreous hemorrhages. There is a vicious cycle of retinal inflammation, hypoxia and neovascularization $[1,2]$. It mostly affects healthy young men, aged $20-40$ years and is a diagnosis of exclusion. It has been reported from the United Kingdom, the United States, and Canada in the latter half of 19th and early twentieth century. However, it is more commonly reported from the Indian subcontinent. The reported incidence in India is 1 in 135 ophthalmic patients in a referral ophthalmic center and 1 in 200 to 250 in a general eye hospital [3]. Since it has been classically described as peripheral vasculitis, macular changes have been reported infrequently in literature. Macular findings have been reported more in recent literature since the advent of better imaging techniques. Some studies have reported that macular changes occur in approximately $18 \%$ of eyes with Eales disease, the most common of which is macular edema. Intravitreal anti vascular endothelial growth factor (VEGF) has been used for treatment of macular oedema. Vitrectomy with epiretinal membrane (ERM) peel has been described for ERM in Eales disease [2, 4-8].

Optical Coherence Tomography Angiography (OCTA) is a novel modality used to study the retinochoroidal vasculature in detail. It is non-invasive and does not require application of intravenous dye. It uses motion contrast imaging to high-resolution volumetric blood flow information generating angiographic images within seconds. OCTA compares the decorrelation signal (differences in the backscattered OCT signal intensity or amplitude) between sequential Optical coherence tomography (OCT) b-scans taken at precisely the same cross-section in order to construct a map of blood flow. Pooling or staining does not occur in OCTA, since there is no dye leaking from pathological microvasculature. This allows better visualization of the whole microvasculature including the foveal avascular zone (FAZ). It has also been reported that deep capillary plexus changes are demonstrated on OCTA but FA is not able to demonstrate these changes $[9,10]$.

India has a high incidence of Eales disease as mentioned above. Since there is infrequent reporting of macular findings in these patients, we conducted this study. There is also scarcity of data in literature about the OCT and OCTA findings of these patients. Our present study throws light on importance of these imaging techniques in Eales disease [8].

\section{Methods}

A prospective cross-sectional study was done on treatment naïve 31 eyes of 23 patients with Eales disease presenting to retina clinic of tertiary care hospital. Informed consent was obtained from all the patients. Permission for conducting the study was obtained from Institutional review board.

Demographic profile of the patient such as age, gender and eye involved was noted. Baseline parameters such as Best-corrected visual acuity (BCVA), slit-lamp bio microscopy (SLB), intraocular pressure and indirect ophthalmoscopy (IO) were done.

Peripheral retinal periphlebitis of small and large caliber vessels with peripheral capillary nonperfusion, and/ or neovascularization elsewhere/of the disc with secondary fibrovascular proliferation on FA was described as peripheral vasculitis. Other findings such as vascular sheathing, perivascular exudates and superficial retinal hemorrhages were noted. All other causes of retinal vasculitis were excluded.

Systemic diseases causing similar presentation such as diabetic retinopathy, vascular occlusion, raised serum homocysteine levels, Sarcoidosis, Syphilis, blood dyscrasias, Coats disease, Familial exudative vitreoretinopathy (FEVR), Acquired immunodeficiency syndrome, Behcet's disease and Sickle-cell disease were excluded. All relevant investigations such as- Complete haemogram with erythrocyte sedimentation rate, peripheral smear, Venereal disease research laboratory test, serum angiotensin converting enzyme levels, Human Immunodeficiency Virus, Hepatitis C Virus, Hepatitis B surface antigen, blood sugar level, serum calcium level, Mantoux test and $\mathrm{X}$-ray chest were done.

Fundus fluorescein angiography (FA) (VISUPAC and FF450plus, Carl Zeiss Meditec, Inc., Dublin, California, USA.) was done. All the relevant findings such as peripheral retinal periphlebitis, peripheral capillary non perfusion, foveal avascular zone abnormalities, and neovascularization elsewhere and of the disc, collateral formation was noted. Macular Ischemia on fluorescein angiography was defined as enlargement of the FAZ and/or capillary dropout encroaching on the FAZ.

Spectral domain- optical coherence tomography (RS3000 Nidek Gamagori, Japan) was used to study the macular changes. ETDRS grid includes 9 quadrants within $6 \mathrm{~mm}$ of foveal center formed by dividing this area by $1 \mathrm{~mm}$ and $3 \mathrm{~mm}$ distance concentric circles. This was used for central macular thickness (CMT) quantitative measurement. Qualitative changes including retinal thickening, cystoid macular oedema, hard exudates, ERM, neurosensory detachment and intra or sub retinal bleed were noted. The data was differentiated into normal or abnormal based on comparison with age matched data of normal patients. 
Optical coherence tomography angiography (RS-3000 Advance, Nidek Gamagori, Japan) was performed to study superficial retina, deep retina, retinal pigment epithelium (RPE) level and choroid. Superficial and deep retinal plexus morphology was studied in detail. Quantitative measurement of superficial retinal capillary plexus (SCP) and deep capillary plexus (DCP) area on OCTA was done.

All the findings on FA, OCT and OCTA were evaluated and reported by two independent observers (KR and MT). The findings were noted to be reproducible, especially OCT and OCTA since they could be repeated every visit.

Macular involvement definition: Macular involvement was defined on FA as macular leakage, enlarged FAZ, capillary dropout at FAZ or epiretinal membrane. Macular involvement on OCT was defined in the form of changes including retinal thickening, cystoid macular oedema, hard exudates, ERM, full thickness macular hole (FTMH), neurosensory detachment and intra or sub retinal bleed at macula.

Patients were classified according to Eales' disease classification system [7]. (Eur J Ophthalmol 2004; 14: 236-9):

Stage 1a: Periphlebitis of small caliber vessels with superficial retinal hemorrhages

Stage 1b: Periphlebitis of large caliber vessels with superficial retinal hemorrhages

Stage 2a: Peripheral capillary non-perfusion

Stage 2b: Neovascularization elsewhere/

Neovascularization of the disc

Stage 3a: Fibrovascular proliferation

Stage 3b: Vitreous hemorrhage

Exclusion criteria:

1. Any media opacity precluding the above investigations, especially OCTA which requires good media clarity, was excluded.

2. Stage 4 disease patients according to Eales' disease classification system [7] were excluded from the study in view of poor visualization of retinal layers (in most of the presenting cases) which is essential for OCT and OCTA.

Outcome measures included:

Primary:

1. Comparison of mean Logmar BCVA among patients with macular changes in FA, OCT and OCTA.

2. Calculation of SCP and DCP area and its correlation with macular changes on OCT.
Secondary:

1. Comparison of qualitative changes on FA, OCT and OCTA in macula.

2. Comparison of CMT on OCT in patients with macular involvement and those without macular involvement.

3. Qualitative changes on OCT in patients with macular involvement and comparison with those without macular involvement.

4. Qualitative changes on OCTA in patients with macular involvement and comparison with those without macular involvement.

Statistical analysis was done using SPSS 16 (SPSS Inc., Chicago, Illinois, USA). The data was presented as mean \pm standard deviation. Mann Whitney $U$ test was used for analysis of quantitative variables such as BCVA, CMT and superficial and deep capillary plexus area between the groups. $P<0.05$ was considered statistically significant.

\section{Results}

Thirty-one eyes of 23 consecutive patients with Eales disease were included.

\section{Demography}

Baseline characteristics of study population are given in Table 1. The baseline Logmar BCVA ranged from 0.0 to 1.8 (Mean: $0.5 \pm 0.43$ ).

Seven patients $(35 \%)$ had a family history of tuberculosis in close family members living with them. Three patients were known cases of tuberculosis on antitubercular treatment.

Sixteen $(51.6 \%)$ patients had macular changes as seen on all modalities together. SLB and IO missed the finding in 8 patients $(50 \%)$ out of total patients with macular involvement. FA missed macular findings in 3 (18.8\%) patients. OCT and OCTA demonstrated the findings in $100 \%$ patients (Table 2 ).

Table 1 Baseline characteristics of patients

\begin{tabular}{ll}
\hline Total eyes & 31 \\
Mean age & $24.87 \pm 5.7$ years (17 to 36 years) \\
Gender & \\
$\quad$ Male & 27 male (87.1\%) \\
$\quad$ Female & 4 female \\
Bilateral involvement & 8 patients \\
Known history of Tuberculosis & 3 patients (13\%) \\
Family history of Tuberculosis & 7 patients (30.4\%) \\
BCVA & 0.0 to 1.8 (Mean: $0.5 \pm 0.43$ ) \\
\hline
\end{tabular}


Table 2 Number of patients with macular changes

\begin{tabular}{ll}
\hline Total patients with macular changes & 16 patients (51.6\%) \\
$\begin{array}{l}\text { Patients with macular changes on SLB and } \\
\text { indirect ophthalmoscopy }\end{array}$ & 8 patients (50\%) \\
Patients with macular changes on FA & 13 patients (81.25\%) \\
Patients with macular changes on OCT and OCTA & 16 patients (100\%) \\
\hline
\end{tabular}

Other than ischemic changes at macula, FA also demonstrated changes such as leakage at macula, window defect in FTMH, peripheral periphlebitis, peripheral capillary non perfusion and neovascularization elsewhere and of the disc.

Classification of cases according to Eales' disease classification system [7] (Eur J Ophthalmol 2004; 14: 2369): The classification of cases is given in Table 3. Stage $2 \mathrm{~b}$ had the highest number of cases and also maximum cases with macular involvement. Due to the small number of cases in each group, intergroup comparison was not done (Fig. 1).

\section{Primary outcome}

The comparison of eyes with macular involvement on FA, OCT and OCTA in terms of mean Logmar BCVA was done (Table 4).

We compared the BCVA between the eyes with macular involvement on FA versus eyes with no involvement of macula on FA. The $p$ value was statistically significant $(p=0.000)$. Thus, FA was able to accurately predict macular changes and corresponding visual acuity reduction. We also compared the mean BCVA between the eyes with macular involvement on OCT versus eyes with no involvement of macula on OCT. The $\mathrm{p}$ value was statistically significant $(p=0.01)$.

On comparison of eyes with macular involvement on OCTA versus eyes with no involvement of macula on OCTA with mean BCVA, eyes with involvement had significantly lower BCVA than those with normal macula (p 0.001).

Table 3 Classification of cases according to Eales' disease classification system. (Eur J Ophthalmol 2004; 14: 236-9)

\begin{tabular}{ll}
\hline Stages & Number of cases \\
\hline Stage 1a & 2 patients (1 patient with macular involvement) \\
Stage 1b & 1 patients (No macular involvement) \\
Stage $2 \mathrm{a}$ & 3 patients (2 patients with macular involvement) \\
Stage $2 \mathrm{~b}$ & 19 patients (7 patients with macular involvement) \\
Stage 3a & 2 patients (1 patients with macular involvement) \\
Stage 3b & 4 patients (2 patients with macular involvement) \\
\hline
\end{tabular}

OCTA findings and SCP-DCP correlation with macular changes on OCT

It was noted in all the patients that only the retinal layers were involved on OCTA with sparing of RPE and choroid. In patients with FTMH and sub-ILM bleed, back shadowing was noted (Figs. 2 and 3). Average superficial and deep capillary plexus areas are given in Table 5.

On performing statistical analysis, the correlation of superficial plexus area with the macular involvement was not statistically significant (p 0.12). However, the deep plexus area and macular involvement was significantly positively correlated ( $\mathrm{p} 0.00$ ).

\section{Secondary outcomes}

Table 6. Data of patients with macular involvement on FA, OCT and OCTA. Comparison of the findings on these three modalities is given for all the individual patients.

\section{Central macular thickness on OCT}

Mean central macular thickness was $290.53 \pm 48.28$. Range was 191 to $395 \mu$. Mean CMT in eyes with macular involvement was $306.83 \pm 62.10 \mu$. Mean CMT in eyes without macular involvement was $279.7 \pm 34.18 \mu$. On comparison, the CMT in eyes with macular involvement was higher than eyes with no macular involvement but this finding was not statistically significant (p 0.09).

The commonest macular finding on OCT was diffuse retinal thickening. Other findings noted were cystoid macular oedema, sub-foveal detachment, epiretinal membrane, retinal thinning, hard exudates at macula, sub-internal limiting membrane bleed and FTMH.

\section{Comparison of foveal avascular zone on FFA and OCTA}

We studied the foveal avascular zone in detail on FFA and OCTA. Any discrepancy between the two was noted. We observed that distorted FAZ on FA suggestive of ischemic maculopathy was seen in 5 patients. However, on OCTA, distorted superficial capillary plexus FAZ was seen in 3 patients and distorted deep capillary plexus FAZ was seen in 11 patients.

\section{Discussion}

Our present study highlights the presence of macular changes as a common finding in Eales disease patients presenting to a tertiary care eye hospital. Macular involvement has been previously described in literature. In a study conducted in patients with Eales disease between 1989 and 1997, on SLB and FA, macular involvement was found in $28 \%$ of cases. The same authors, in a retrospective analysis, reported 


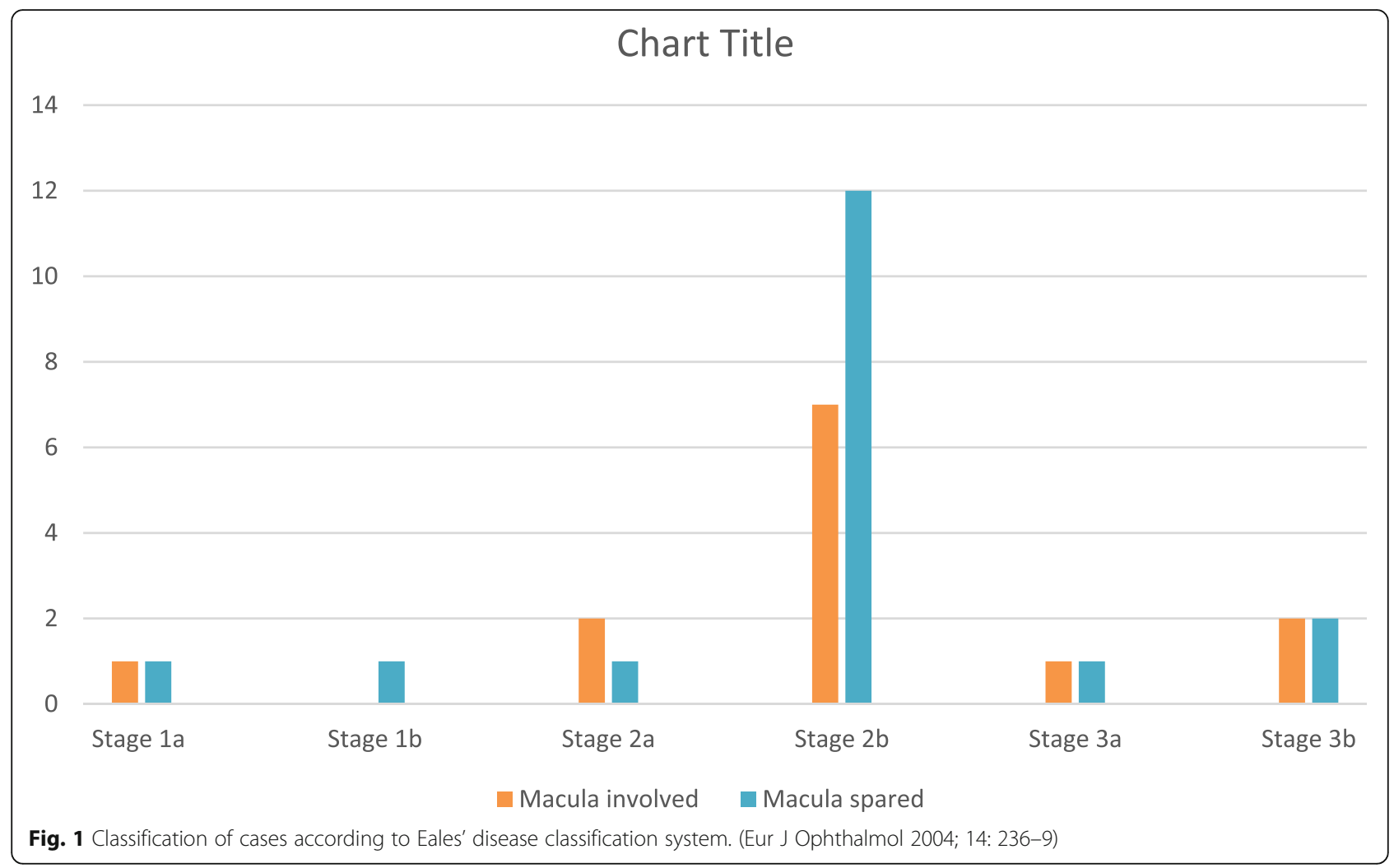

macular involvement seen in 32\% cases in patients with Eales disease. Another study has reported macular involvement in $35.4 \%$ patients [11]. A recent study reported that $58.2 \%$ eyes of Eales disease patients had macular involvement as assessed with SD-OCT [8]. Thus, they noted macular changes to be a frequent finding. Macular oedema was the most common (35.4\%) followed by ERM (11.4\%) and macular thinning (11.4\%). Other macular changes reported were hard exudates $(6.3 \%)$, macular hemorrhages (5\%), internal retina or internal limiting membrane folds (3.8\%), pre macular hemorrhages (2.5\%) and macular holes (1.2\%). It has been reported that macula is usually not involved primarily in Eales disease despite extensive peripheral non-perfusion. But when it does get involved, it is termed central Eales disease.
In this condition, all the classic mid-peripheral lesions appear at the posterior pole and cause decreased vision in the early stage of the disease, often due to cystoid macular oedema [12]. 51.6\% of our patients had macular changes as seen on all modalities together. Many of our patients had classic Eales lesions at macula, thus suggestive of central Eales.

Macular changes in Eales disease have been increasingly reported in recent literature. We feel that one of the reasons for this is the advent of newer modalities like OCT and OCTA which give exact enface and crosssectional images of the retina.

The age range reported in our study is in accordance with other studies which report the age range to be between 20 to 40 years $[8,12]$. The male preponderance

Table 4 Comparison of mean BCVA among patients with or without macular involvement on FA, OCT and OCTA

\begin{tabular}{|c|c|c|c|c|}
\hline IMAGING MODALITY & Macular involvement & Number of patients & BCVA & $P$ value \\
\hline \multirow[t]{2}{*}{ FA } & Macula involved & 10 patients & $0.87 \pm 0.44$ (0.48 to 1.8 ) & 0.000 \\
\hline & Macula non-involved & 21 patients & $0.33 \pm 0.31$ (0.0 to 1.18 ) & \\
\hline \multirow[t]{2}{*}{ OCT } & Macula involved & 13 patients & $0.7 \pm 0.48$ (0.0 to 1.8$)$ & 0.01 \\
\hline & Macula non-involved & 18 patients & $0.36 \pm 0.33$ (0.0 to 1.8$)$ & \\
\hline \multirow[t]{2}{*}{ OCTA } & Macula involved & 12 patients & $0.8 \pm 0.46(0.3$ to 1.8$)$ & 0.001 \\
\hline & Macula non-involved & 19 patients & $0.32 \pm 0.3(0.0$ to 1.18$)$ & \\
\hline
\end{tabular}





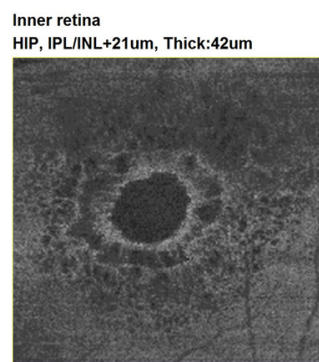

DCP

DCP
AIP, IPLINL+13um to IPLIINL+88um

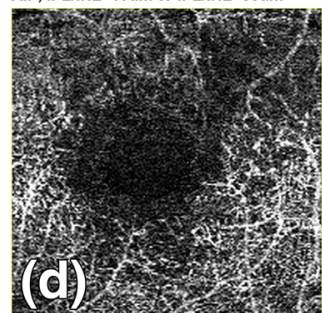

RPE

RPE

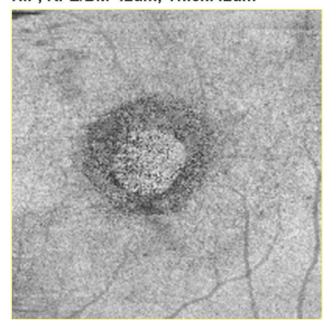

Outer retina

IPLINL+92um to RPE/BM surface fit+0um

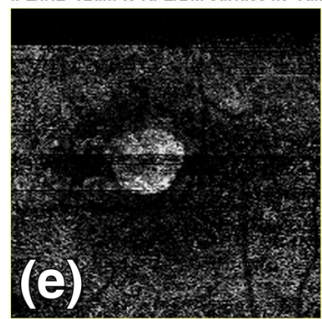

Choroid

HIP, RPE/BM+0um, Thick:126um

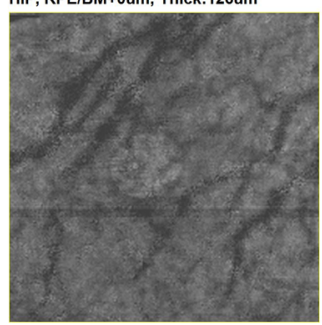

Choroid

AIP, RPE/BM+4um to RPE/BM+63um

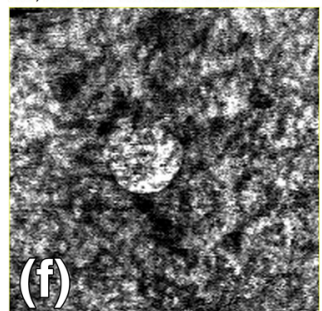

Fig. 236 years old male with vision in left eye counting finger 3 m; (a) FA: window defect at macula due to FTMH (b) OCT: shows FTMH with cuff of fluid (c) OCTA at SCP (d) OCTA at DCP (e) OCTA at outer retina (f) OCTA at choroid level shows FTMH with back shadowing

$(87.1 \%)$ as seen in our study, has been reported previously [3].

The comparison of mean BCVA in patients with and without macular involvement diagnosed on FA, OCT and OCTA has scarcely been done before. We noted that the mean BCVA was less in patients with macular changes compared to those without macular changes $(p<0.05)$. This has been previously reported [8]. Though the $p$ value was significant on all three modalities separately, however, FA missed some macular involvement patients which were picked up on OCT and OCTA. Thus, OCT and OCTA are very sensitive tools for analysis of macular changes in Eales disease and also predictors of visual acuity.
OCTA of Eales disease patients has scarcely been done [10]. In our study, SCP and DCP area was enlarged on OCTA in patients with macular involvement. The deep plexus area and macular involvement was highly significantly correlated (p $0.00)$. We concluded that the deep capillary plexus enlargement has more association with foveal involvement. The FAZ area calculation in Eales disease patients has never been reported before and since normative database of Indian eyes is not yet available, comparison of our data with normal could not be done. We also noted that RPE and choroid level OCTA scans were normal, thus suggestive of a retinal vascular pathology since it affected only the retinal layers. 


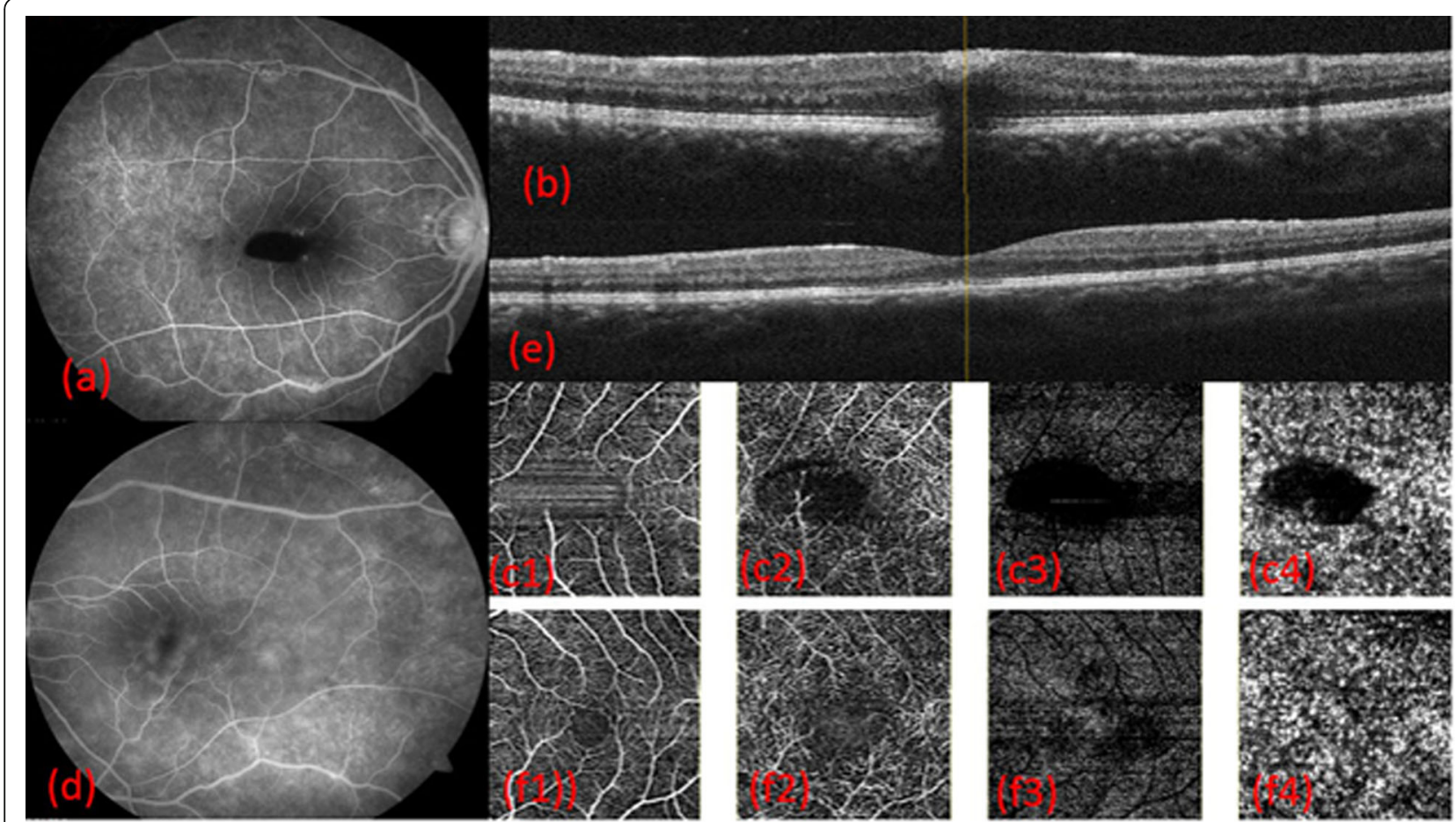

Fig. 317 years male vision right eye counting finger $1 \mathrm{~m}$ and left eye 6/24; (a) FA: Right eye with blocked fluorescence (b) OCT: shows sub-ILM bleed (c1-4) OCTA at SCP, DCP, outer retina and choroid shows back-shadowing due to bleed (d) FA: Left eye with leakage at macula (e) OCT shows outer retina mild irregularity (f1-4) OCTA at SCP,DCP, outer retina and choroid level suggestive of DCP enlargement

It has been demonstrated that OCTA can be a better modality for macular changes, especially in the perifoveal area, than FA, since it distinguishes the different layers of retina and choroid, which cannot be done on FA. It also differentiates the superficial from deep plexus which is not possible on FA. Quantification of capillary plexuses can be done for follow up on OCTA and also visual prognostication. Pooling and leakage which can hamper macular analysis are not seen on OCTA thus making it a good modality for macular analysis. The adverse events related to dye injection are also averted on OCTA. However, periphery evaluation for neovascularization and capillary non-perfusion needs FA since it has a wider field of view $[9,10]$.

Table 5 SCP-DCP area

\begin{tabular}{ll}
\hline RETINAL PLEXUS ON OCTA & AVERAGE AREA OF PLEXUS \\
\hline SCP & $0.39 \pm 0.13$ (Range 0.18 to 0.79) $\mu$ \\
$\begin{array}{l}\text { SCP area in eyes with macular } \\
\text { involvement }\end{array}$ & $0.45 \pm 0.14$ (Range 0.28 to 0.79) $\mu$ \\
DCP & $0.69 \pm 0.23$ (Range 0.31 to 1.49) $\mu$ \\
$\begin{array}{l}\text { DCP area in eyes with macular } \\
\text { involvement }\end{array}$ & $0.88 \pm 0.24$ (Range 0.65 to 1.49) $\mu$. \\
\hline SCP Superficial capillary plexus; DCP Deep capillary plexus area
\end{tabular}

In our study, SLB and IO missed the finding in 8 patients $(50 \%)$ out of the total patients with macular involvement. In another study [8], 28.3\% were noted to have no macular changes based on SLB though they were noted on OCT. FA missed macular findings in 3 (18.8\%) patients in our study. OCT and OCTA demonstrated the findings in $100 \%$ patients.

We noted that FA was able to identify vasculitis and leakage at macula accurately. However, macular changes in the form of capillary non perfusion, distorted FAZ especially at the deep capillary plexus were missed in 6 out of total 11 patients on FA. One patient with unexplained loss of vision with normal SLB and FA actually showed changes in DCP which explained the visual loss. DCP enlargement disproportionate to SCP enlargement was noted in 8 patients. These patients also had visual loss, thus, highlighting the importance of DCP involvement in Eales disease patients and its effect on their vision (Figs. 4 and 5). There have been previous reports highlighting the changes in DCP selectively in retinal venous occlusion corresponding with vision. It has also been reported that FA can identify SCP changes but not DCP changes separately since it is a two dimensional imaging modality [13-18].

We classified our cases according to Eales' disease classification system [7] and observed that all the 
Table 6 Data of patients with macular involvement on FA, OCT and OCTA

\begin{tabular}{|c|c|c|c|c|c|c|c|}
\hline No. & Age (years) & Gender & $B C V A$ & Changes in macula on FA & CMT & OCT & OCTA \\
\hline 1. & 17 & M & 0.3 & Sub ILM bleed-blocked fluorescence & 368 & Sub-ILM bleed & Back shadowing of bleed \\
\hline 2. & 20 & M & 1 & WNL & 308 & WNL & Enlarged deep capillary plexus \\
\hline 3. & 22 & M & 0.48 & Ischemic macula & 260 & Few cystoid spaces & Enlarged deep capillary plexus \\
\hline 4. & 23 & M & 0.6 & Ischemic macula & 307 & Diffuse retinal thickening & Distorted plexus \\
\hline 5. & 23 & M & 0.78 & Ischemic macula & 282 & Diffuse retinal thickening & Distorted plexus \\
\hline 6. & 22 & M & 0.6 & Distorted macular vessels, ERM & 226 & $\begin{array}{l}\text { ERM blunted foveal contour, retinal } \\
\text { thinning }\end{array}$ & Distorted plexus \\
\hline 7. & 24 & M & 0.48 & Ischemic macula & 394 & CME and NSD & Enlarged deep capillary plexus \\
\hline 8. & 21 & M & 0.3 & WNL & 325 & Diffuse retinal thickening & Enlarged deep capillary plexus \\
\hline 9. & 18 & $\mathrm{~F}$ & 1.3 & Ischemic macula & 303 & $\begin{array}{l}\text { Diffuse oedema in inferotemporal } \\
\text { quadrant, hard exudates }\end{array}$ & Enlarged deep capillary plexus \\
\hline 10. & 36 & M & 1.3 & Window defect & FTMH & FTMH & Enlarged deep capillary plexus \\
\hline 11. & 17 & M & 0.6 & Leakage at macula & 282 & WNL & Enlarged deep capillary plexus \\
\hline 12. & 35 & M & 0.3 & WNL & 283 & WNL & Enlarged deep capillary plexus \\
\hline 13. & 25 & M & 0.6 & WNL & 395 & CME & WNL \\
\hline 14. & 32 & M & 0 & WNL & 321 & Diffuse retinal thickening & WNL \\
\hline 15. & 25 & M & 0.3 & WNL & 310 & Diffuse retinal thickening & WNL \\
\hline 16. & 19 & $M$ & 0.78 & Ischemic macula, leakage at macula & 191 & Hard exudates at macula & WNL \\
\hline
\end{tabular}

M Male; F Female; VN: Vision; CMT Central macular thickness; WNL Within normal limit; FTMH Full thickness macular hole; ERM Epiretinal membrane; ILM Internal limiting membrane; NSD Neurosensory detachment

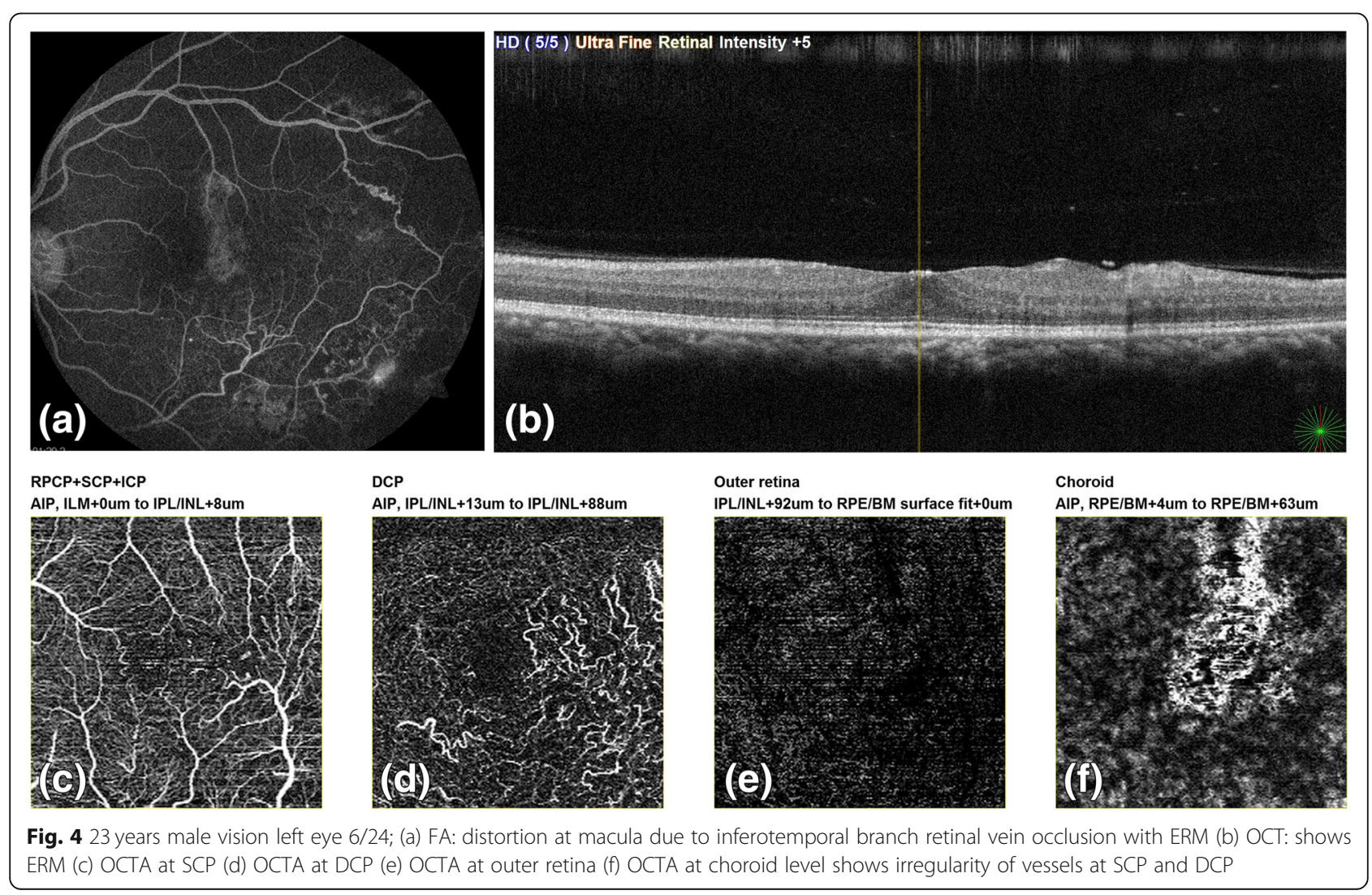




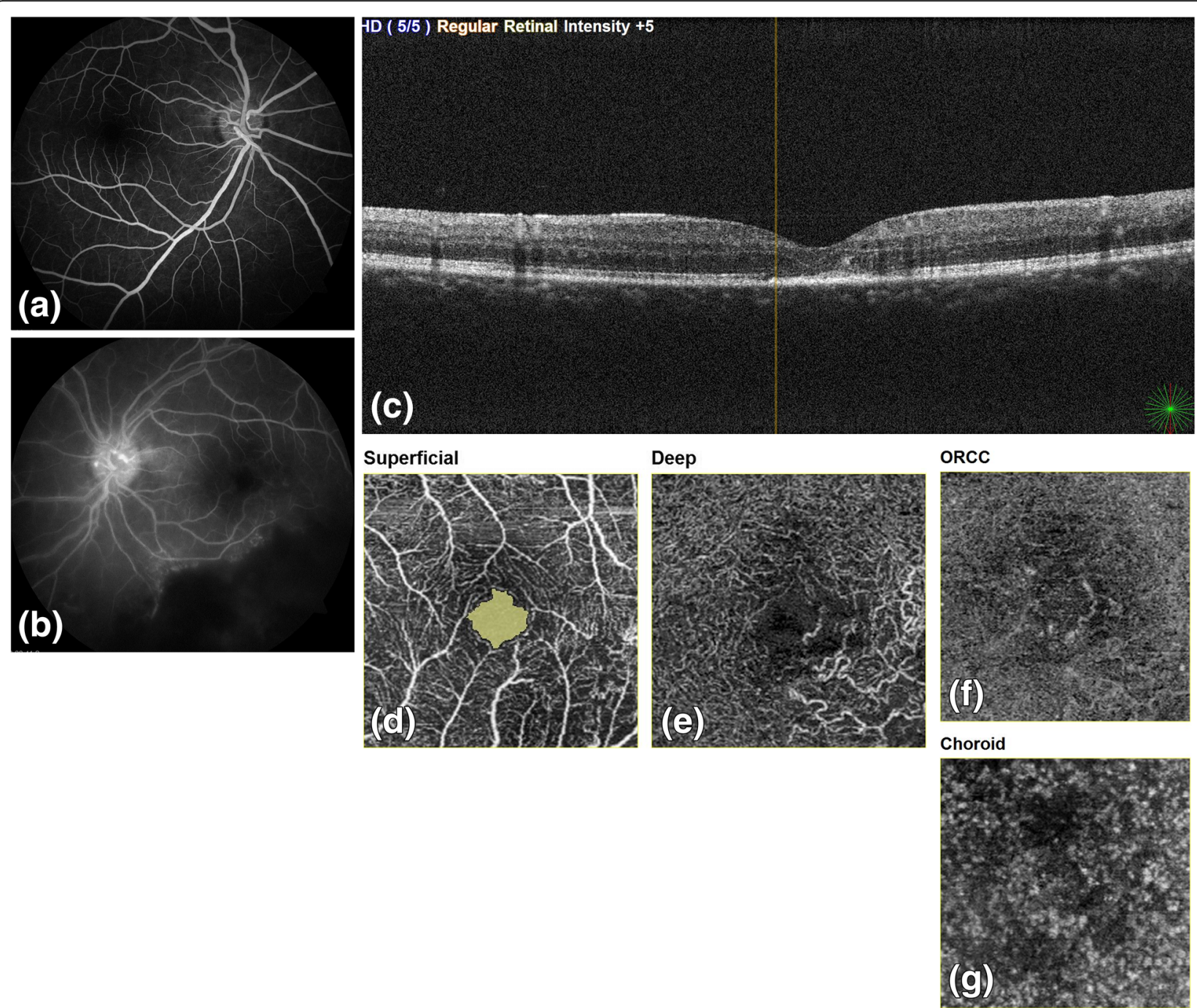

Fig. 522 years male vision left eye 6/18; (a) FA right eye: WNL (b) FA left eye macula appears WNL, capillary non perfusion encroaching posterior pole; FAZ appears normal (c) OCT: Few cystoid spaces and mild distortion of outer retinal layers (d) OCTA at SCP shows manually marked area of SCP: WNL (e) OCTA at DCP which is enlarged (f) OCTA at outer retina (g) OCTA at choroid level

groups except stage $1 \mathrm{~b}$ had macular involvement. Stage $2 b$ had the highest number of macular involvement patients.

On comparison of CMT in eyes with macular involvement versus eyes without macular involvement, CMT of eyes with macular involvement was higher than eyes without it, but this finding was not statistically significant ( $p$ 0.09). This is in accordance with other studies [8]. The qualitative macular changes noted on OCT were diffuse retinal thickening, cystoid macular oedema, sub-foveal detachment, epiretinal membrane, retinal thinning, hard exudates at macula, sub-internal limiting membrane bleed and FTMH. Macular oedema as the commonest finding in Eales has been reported recently $[7,8,11,19]$. Other changes have scarcely been reported, especially FTMH $[8,19]$. Premacular haemorrhage and retinal thinning are also rare [8].

Our study included multimodal analysis of macular changes in Eales disease with SLB, IO, FA, OCT and OCTA which has never been done before. This, we feel, is the strength of our study. Our limitation is the small number of patients included. Eales disease being a rare entity and a diagnosis of exclusion, it is difficult to conduct large sample studies on these patients. Some patients in whom media is not clear, the above investigations cannot be conducted. This fact limits the number of study patients. A larger study may be able to establish the results demonstrated by our pilot study. 


\section{Conclusion}

Eales disease though described in literature as classically being a peripheral retina disease process, also has macular involvement especially in patients with central fundus findings and neovascularization. Macular oedema is the commonest macular abnormality in these patients. Macular involvement is associated with decreased visual acuity. Hence, it should be actively looked for in Eales disease. OCT and OCTA are useful guides to evaluation of macula and visual prognostication. OCTA, which is non-invasive, seems to be superior to FA in detecting macular abnormalities in this ailment. FAZ measurement for comparison can also be done with OCTA. It also shows DCP changes which are not shown by any other modality. In view of these advantages, OCT and OCTA should be routinely recommended for all Eales disease patients.

\section{Abbreviations \\ OCT: Optical coherence tomography; OCTA: Optical coherence tomography angiography; SLB: Slit lamp bio microscopy; IO: Indirect ophthalmoscopy; FA: Fluorescein angiography; FAZ: Foveal avascular zone; CMT: Central macular thickness; SCP: Superficial capillary plexus; DCP: Deep capillary plexus; LOGMAR BCVA: Logarithm of minimum angle of resolution best corrected visual acuity; ERM: Epiretinal membrane; RPE: Retinal pigment epithelium}

\section{Acknowledgements}

Not applicable.

\section{Authors' contributions}

MT conceptualized and designed the study. All the data collection was done by KR and PG. Analysis of the data was done by KR and interpretation was done by MT and KR. Literature search was done by PG. AR was involved in making finer changes in the manuscript and approving the final version of it. Each author has agreed both to be personally accountable for the author's own contributions and to ensure that questions related to the accuracy or integrity of any part of the work, even ones in which the author was not personally involved, are appropriately investigated, resolved, and the resolution documented in the literature. All the authors have read and approved the final submitted version of the manuscript.

\section{Authors' information}

None.

\section{Funding}

Not applicable.

\section{Availability of data and materials}

The datasets used and/or analyzed during the current study are available from the corresponding author on reasonable request.

\section{Ethics approval and consent to participate}

Institutional ethics approval and consent from all the participants was obtained.

\section{Consent for publication}

Not applicable.

\section{Competing interests}

The authors declare that they have no competing interests.
Received: 25 April 2020 Accepted: 15 October 2020

Published online: 14 December 2020

\section{References}

1. Patnaik B, Nagpal PN, Namperumalsamy P et al (1998) Eales disease: clinical features, pathophysiology, etiopathogenesis. Ophthalmol Clin N Am 11:601-618

2. Biswas J, Sharma T, Gopal L et al (2002) Eales disease - an update. Surv Ophthalmol 47:197-214

3. Das T, Biswas J, Kumar A et al (1994) Eales' disease. Indian J Ophthalmol 42: $3-18$

4. Agrawal S, Agrawal J, Agrawal T (2006) Intravitreal triamcinolone acetonide in Eales' disease. Retina 26:227-229

5. Majji AB, Vemuganti GK, Shah VA et al (2006) A comparative study of epiretinal membranes associated with Eales' disease: a clinicopathologic evaluation. Eye 20:46-54

6. Gupta SR, Flaxel CJ (2012) The use of a vascular endothelial growth factor inhibitor (ranibizumab) in macular edema due to eales disease. Retinal Cases Brief Rep 6:122-124

7. Saxena S, Kumar D (2007) New classification system-based visual outcome in Eales' disease. Indian J Ophthalmol 55:267-269

8. Goel N, Kumar V, Arora S et al (2018) Spectral domain optical coherence tomography evaluation of macular changes in Eales disease. Indian J Ophthalmol 66:433-438

9. de Carlo TE, Romano A, Waheed NK (2015) A review of optical coherence tomography angiography (OCTA). Int J Retina Vitreous 1:5

10. Abucham-Neto JZ, Torricelli AAM, Lui ACF et al (2018) Comparison between optical coherence tomography angiography and fluorescein angiography findings in retinal vasculitis. Int J Retin Vitr 4:15

11. Saurabh K, Das RR, Biswas J (2011) Profile of retinal vasculitis in a tertiary eye care center in eastern India. Indian J Ophthalmol 59:297-301

12. Das T, Pathengay A, Hussain N (2010) Eales' disease: diagnosis and management. Eye 24:472-482

13. Albalkini AS, El-Sanabary Z, Khalil NM (2019) Imaging of retinal vein occlusion by optical coherence tomography-angiography (OCTA). J Eye Stud Treat 1:40-50

14. Suzuki N, Hirano Y, Yoshida M et al (2015) Microvascular abnormalities on optical coherence tomography angiography in macular edema associated with branch retinal vein occlusion. Am J Ophthalmol 161:126-132

15. Martinet V, Guigui B, Glacet-Bernard A et al (2012) Macular optical coherence tomography, fluorescein angiography and visual acuity. Int Ophthalmol 32:369-377

16. Bonnin S, Mane V, Couturier A et al (2015) New insight into the macular deep vascular plexus imaged by optical coherence tomography angiography. Retina 35:2347-2352

17. Tan PE, Yu PK, Balaratnasingam C et al (2012) (2012) quantitative confocal imaging of retinal microvasculature in the human retina. Invest Ophthalmol Vis Sci 53:5728-5736. https://doi.org/10.1167/iovs.12-10017

18. Browning DJ (2012) Pathophysiology of retinal vein occlusions. In: Browning DJ (ed.). Retinal vein occlusions: evidence-based management. Springer, New York. 2: 33-72

19. Saxena S, Kumar D (2000) Macular involvement in Eales disease. Ann Ophthalmol 32:98-100

\section{Publisher's Note}

Springer Nature remains neutral with regard to jurisdictional claims in published maps and institutional affiliations. 